Article

\title{
Evaluation of thiol-dependent enzymes on the pharmacological effects induced by the catalytically active PLA2 from Bothrops
} jararacussu

\author{
Marcos Hikari Toyama1*, Caroline R. C. Costa ${ }^{1}$, Mariana Novo Belchor ${ }^{1}$, Adeilso Bispo dos Santos Junior ${ }^{1}$, Laila \\ Lucyane Ferreira de Moraes ${ }^{1}$, Airam Roggero dos Santos Silva ${ }^{1}$ and Marcos Antonio de Oliveira ${ }^{2}$.
}

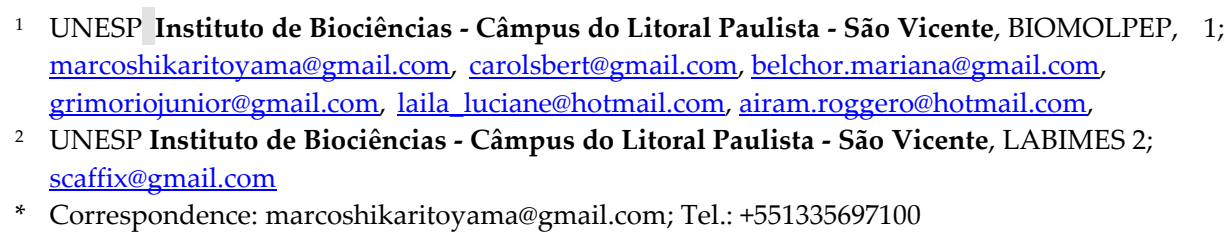

\begin{abstract}
Background: Clinical cases reports with snake accidents show that venom bite induces increased oxidative stress including several markers of lipid peroxidation and other oxidative stress marker in plasma. Methods: The main findings of this work were performed with BthTx-II on paw edema of animals treated with the toxin and biochemical measurement of COX-2, PGE2, MDA and the effects of peroxiredoxin inhibitors on edema and myotoxicity were also evaluated. Results: The results show that edema and myotoxocity induced by PLA2 (BthTx-II) induces a strong mobilization of arachidonic acid and an increase in cellular oxidative stress as measured by increased malondialdehydo (MDA) concentration and protein carbonylation. Thus, these findings establish the strong link between oxidative stress, arachidonic acid mobilization and that these events may explain the presence of oxidative stress markers in snake-bitten patients. Experiments performed with animals previously treated with commercially purchased inhibitors showed enzymes such as thioredoxin (TXN), thioredoxin reductase (TXNRD) and other glutathione (GSH)-related antioxidant defenses could play an essential role controlling and defining the end of edema on the late phase of PLA2 BthTx-II-induced process. Conclusion. This study showed that thioate-dependent antioxidant enzymes play an important role in resolving the edema induced by BthTx-II.
\end{abstract}

Keywords: secretory phospholipase A2, Bothrops jararacussu, oxidative stress, edema, myonecrosis and thiol dependent antioxidant.

\section{Introduction}

Snakebite is a complex neglected health problem, and the greatest treatment consists of the antivenom therapy, mainly preventing or reversing the snakebite effects through the neutralization of their systemic effects and local manifestations. Furthermore, studies also indicate that during envenomation, cellular and plasma oxidative stress increases when patients arrive at the hospital and this increase in cellular and plasma oxidative stress would play an essential role in the pathophysiological process of envenomation [1-3]. Moreover, some studies have also shown that polyvalent antivenoms administration induces more oxidative stress and lead to the activation of the complement system C3, C4, and C5. The complement system fragments are produced in this process, leading to chemotaxis, neutrophil activation, basophil, and mast cell degranulation, releasing active mediators, which possibly increase oxidative stress in addition to strongly contributing to the development of a larger inflammatory and degenerative process $[2,4,5]$. 
In contrast, the inflammatory and myotoxic action induced by snake PLA2 can be described as two interrelated and linked events between the pro-inflammatory mediators production such as arachidonic acid (AA). The AA releasing leads to pro-inflammatory cascade activation, the reactive oxygen species production, and an increased cellular oxidative stress. These events can be evidenced by the presence of several oxidative markers in serum related to lipid peroxidation, which appears to be a crucial event in the induced toxicity of snake venom and can be observed in snakebite patients $[6-8,3]$.

Among the routes involved in thereactive oxygen species generation, one is observed that requires the AA role, leading to the stimulation of NADPH oxidase (NOX) activity. In the presence of certain transition metals such as $\mathrm{Fe} 2+$ or $\mathrm{Cu}+$, this enzyme reduces hydrogen peroxide and gives rise to the hydroxyl radical $(\bullet \mathrm{OH})$, which reacts rapidly with membrane phospholipids [9 - 12]. Membrane lipoxidation can result in membrane structure and fluidity modifications and the hydroxyl radical can also lead to the AA oxidation wich results on the cell death [13-16]. Hence, ROS levels control is crucial to maintain the cellular integrity, especially controlling lipid lipoperoxidation [17].

Due to the ROS toxic potential, organisms have evolved a complex antioxidant system to survive in this oxidative environment. Glutathione (GSH) and thioredoxin (Trx) are considered the main cellular redox systems related to the cellular redox state maintenance. GSH and Trx oxidized forms are continuously recycled through the enzymes glutathione reductase (GR) and thioredoxin reductase (TrxR), which maintain electron flow to the peroxidases. Glutathione peroxidase (GPx) and peroxiredoxins (PRX) correspond to the most efficient peroxidases from the cellular point of view and in according to the literature mammals exhibit six PRX groups, in addition to expanding the PRX1 group into four closely related subgroups (PRX1-4) plus PRX5 and PRX6 [18-22]. Moreover, these enzymes utilize GSH and GSH S-transferase (GST) for reduction and resolution of their oxidized peroxidative cysteines (Cys) [23, 24].

Commercially, there are at least three inhibitors that are known and used to study the thiol-dependent-enzyme systems. Adenantine (ADNT) is a diterpenoid extracted from the plant Isodon adenantha, which exhibits an inhibitory activity against peroxiredoxin 1 (PRDX1) and, at a lower level, against PRDX2. Furthermore, ADNT can also inhibit other proteins such as thioredoxin (TXN) and thioredoxin reductase (TXNRD), which could hinder the PRDX regeneration rate. Besides that, studies reveal that ADNT can inhibit the binding of peroxidase enzymes, highlighting that this compound could be considered a general inhibitor of multiple enzymes and thiol-dependent systems [25, 26]. The compound 2,3-bis (bromomomethyl) quinoxaline-1,4-dioxide (Conoidin A (ConA)) irreversibly inactivates PRX1 and 2 enzyme activity through a covalent bond formation at the enzymes catalytic site. In addition, ConA also experimentally leads to the inhibition of hyperperoxidation of mammalian peroxiredoxin I and II besides to lead an irreversible oxidation of cysteine resolution, which may contribute to further inhibitory activity [27]. Peroxiredoxin 6 (PRX6), a bifunctional enzyme with glutathione peroxidase and phospholipase A (2) (PLA2) activities, has its activity strongly inhibited by 1Hexadecyl-3-trifluoroethyl glycerol-2-phosphomethanol (MJ33) compounds, which are a fluorinated phospholipid analog that inhibits PRX6 activity. Considering the essential role of these enzymes, this study aims to evaluate the edema, myotoxic and anti-erythrocyte activity of the myotoxic sPLA2 from Bothrops jararacussu in the presence of three commercial peroxiredoxin inhibitors. Moreover, we verified the relevance of the thioldependent antioxidant system in the pharmacological process' management induced by this group of myotoxic sPLA2.

\section{Materials and Methods}

\subsection{Purification of Bothrops jararacussu sPLA2 (BthTX-II).}


The purification of sPLA2 catalytically active from Bothrops jararacussu (BthTx-II) total venom occurred in two steps. Total venom $(20 \mathrm{mg})$ was loaded onto a Protein Pack SP 5PW column $(0.78 \mathrm{~cm} 37.0 \mathrm{~cm})$ at a constant flow rate of $1.0 \mathrm{ml} / \mathrm{min}$ and the samples were eluted using a linear gradient of ammonium bicarbonate $(0.05-1.0 \mathrm{M})$. Chromatographic and elution profile was monitored at absorbance of $280 \mathrm{~nm}$. Fractions containing secretory snake venom PLA2 activity were pooled, lyophilized, and stored at $-20^{\circ} \mathrm{C}$. In the next step, approximately, $5 \mathrm{mg}$ of the PLA2 fraction were dissolved in $250 \mathrm{ml}$ of $0.1 \%$ (v/v) trifluoroacetic acid (solvent A). The resulting solution was clarified by centrifugation and supernatant was applied to a m-Bondapak C-18 column $(0.78 \mathrm{~cm} 330 \mathrm{~cm}$ : Waters 991-PDA system). Proteins were eluted with a linear gradient (0-100\%) of $66.5 \%$ $(\mathrm{v} / \mathrm{v})$ acetonitrile in $0.1 \%(\mathrm{v} / \mathrm{v})$ trifluoroacetic acid (solvent $\mathrm{B}$ ) at a flow rate of $2.0 \mathrm{ml} / \mathrm{min}$. The elution profile was monitored at $280 \mathrm{~nm}$ and fractions were collected, lyophilized, and stored at $-20^{\circ} \mathrm{C}$. Protein purity was confirmed by PAGE-SDS and by size exclusion chromatography (SEC).

\subsubsection{Enzymatic activity}

To confirm the sPLA2 enzymatic activity, the enzyme was dissolved in a $0.9 \% \mathrm{NaCl}$ (saline solution) at a final concentration of $1 \mathrm{mg} / \mathrm{mL}$. sPLA2 samples were incubated with the compounds in microplates for $20 \mathrm{~min}$ at room temperature in the presence of a chromogenic substrate for phospholipase (NOBA). The substrate was solubilized in acetonitrile at $1 \mathrm{mg} / \mathrm{mL}$. The compounds were incubated with sPLA2 for $30 \mathrm{~min}$ to evaluate the enzymatic inhibition. Both were added to $0.02 \mathrm{M}$ Tris- $\mathrm{HCl}, 0.15 \mathrm{M} \mathrm{NaCl}$, and $1 \mathrm{mM}$ $\mathrm{CaCl} 2(\mathrm{pH} 8)$ buffer for analysis via a SPECTRA MAX spectrophotometer (Molecular Devices, Sunnyvale, CA, USA) at a wavelength of $425 \mathrm{~nm}$. The measurements were performed throughout the incubation time $(90 \mathrm{~min})$ with five-minute intervals between readings.

\subsection{Pharmacological Assay and Biochemical Assays.}

\subsubsection{Paw Edema.}

Female Swiss mice (20-25 g, $\mathrm{n}=5$ ) were obtained from the Multidisciplinary Centre for Biological Research (CEMIB) of the State University of Campinas (UNICAMP). The animals were maintained under standard conditions $\left(25^{\circ} \mathrm{C}\right.$; 12 -h light/dark cycle) with food and water available ad libitum. In vivo experimental models were performed to evaluate the acute inflammation inhibition induced by purified BthTX-II, using randomly chosen mice (Mus musculus). Animals were treated with $50 \mu \mathrm{L}$ of PRXs' inhibitor which are administered through peritoneal injection on animals 30 minutes prior edema assay. Paw edema was induced by the single subplantar injection of sPLA2 and this procedure was made to evaluate the previous incubation of these inhibitor on the BthTX-II injection. Each site received an injection of $20 \mu \mathrm{L}$ of sample $(0.5 \mathrm{mg} / \mathrm{mL})$; therefore, each site received $10 \mu$ g per paw and saline solution $(0.9 \% \mathrm{NaCl})$ was used as the negative control. In total, 8 groups were included: saline, BthTx-II, BthTx-II + ADNT 30'(Adenanthin), BthTx-II + ConA (Conoidin A) 30', BthTx-II + MJ33 30', ADNT, ConA, MJ33. The monitoring of edema volume was performed using the LE7500 Digital Plethysmometer (Panlab, Harvard Apparatus, Cornellà, Spain) and the results were expressed as edema in microliter and interval time. Paw edema volumes were measured immediately before injection and at selected time intervals afterwards $(0,30,60,120,180,240,300,360,420$ and $480 \mathrm{~min}$ ) Results are expressed as the increase in paw volume (microliters), calculated by initial volume subtraction and each treatment was performed for $n=5$. After the tests, rats were anesthetized and sacrificed by cervical dislocation. In vivo experiments were performed according to the institutional rules and they were approved by ethics committee from UNESP, number 008-CEUA. 


\subsubsection{Biochemical Determination.}

Animals were also used for enzymes quantification carried out with commercial kits. Blood was collected from mice with heparin, before being centrifuged. Plasma was separated and stored at $-80^{\circ} \mathrm{C}$ for use in the COX-2, MDA, protein carbonylation and assays (Abcam, Cambridge, MA, USA). Gastrocnemius muscle from the mouse's right foot was collected and stored at $-80^{\circ} \mathrm{C}$ for prostaglandin E2 (PGE2) quantification.

\subsubsection{Evaluation of COX-2 Levels.}

COX-2 levels were analyzed using an enzyme-linked immunosorbent assay (ELISA) kit (ab210574; Abcam, Cambridge, MA, USA), according to manufacturer's guidelines.

\subsubsection{PGE2 quantification.}

PGE2 were determined using an ELISA kit (ab133021; Abcam, Cambridge, MA, USA), according to manufacturer's guidelines.

\subsubsection{Protein carbonylation Quantification.}

Protein carbonylation was determined by ELISA for quantitative determination of carbonylated protein levels in plasma (ALX-850-312-KI01, Enzo Life Science, New York, NY, USA), according to manufacturer's guidelines.

\subsubsection{Free Thiol Quantification.}

Free thiol content was determined in plasma using MET 5053 (Total Thiol Assay Kit (Colorimetric), Cell Biolabs, Inc., San Diego, CA, USA), according to manufacturer's guidelines.

\subsubsection{Lipid Peroxidation Determination (MDA).}

The extent of lipid peroxidation was determined monitoring MDA levels using an ELISA kit (ab118970; Abcam, Cambridge, MA, USA), according to manufacturer's guidelines.

\subsubsection{Myonecrosis evaluation.}

Myotoxic activity was evaluated through creatine kinase released (CK) on plasma. Damage muscle cells released creatine kinase (CK) and levels were measured using a commercial CK-NAc kit (Laborlab, London, UK). Briefly, in the right gastrocnemius muscle was injected $20 \mu \mathrm{L}$ of BthTx-II sample (at $1 \mathrm{mg} / \mathrm{mL}$ ), whereas control group received an equal volume of $0.15 \mathrm{M} \mathrm{NaCl}$. After 30minutes, the animals were anesthetized, and samples were collected from the abdominal cavity into tubes containing heparin as an anticoagulant. The plasma was stored at $-10^{\circ} \mathrm{C}$ for a maximum of $12 \mathrm{~h}$ before the assay. CK levels were determined with $40 \mu \mathrm{L}$ of plasma, which was incubated for $3 \mathrm{~min}$ at $37^{\circ} \mathrm{C}$ with $1.0 \mathrm{~mL}$ of the reagent according to the protocol kit. Resulting activity is expressed in U/L. Animals were treated with $50 \mu \mathrm{L}$ of PDXs inhibitor which are administered through peritoneal injection on animals 30 minutes prior edema assay that performed on posterior paw oedema was induced by the single subplantar injection of sPLA2 and this procedure it was made to evaluate the previous incubation of these inhibitor on the BthTx-II injection. Each treatment was conducted for $n=5$. After the tests, mice were anesthetized and sacrificed via cervical dislocation. Experiments were performed according to the institutional rules and they were approved by ethics committee from UNESP, number 008-CEUA.

\subsubsection{Treatment of animals with inhibitors}


A total of $50 \mu \mathrm{L}(1 \mathrm{mmol} / \mathrm{mL}$, stock solution) of Adenanthin (ADNT, Chem Faces, Wuhan, Hubei PRC); MJ33 (as the lithium salt, Cayman Chemical Cat No 90001844, Ann Arbor, Michigan, USA) and Conoidin A (ConA, Cayman Chemical Cat No 90001844, Ann Arbor, Michigan, USA) were dissolved in DMSO at first time and diluted in saline to final DMSO concentration of 3\% and $1 \mathrm{mmol} / \mathrm{mL}$. Each inhibitor (ADNT, MJ33 and ConA) was injected intraperitoneally 30 min before the application of BthTx-II. Animals were treated with $50 \mu \mathrm{L}$ of PRXS inhibitor which are administered through peritoneal injection on animals 30 minutes prior edema and myonecrosis. In addition, all negative controls were performed using saline.

2.3. Mouse Red Blood Cells: Hemolysis, Lipid peroxidation and Oxidation.

2.3.1. Isotonic hemolysis.

Mouse Red Blood Cells was supplied as a $10 \%$ suspension of murine red blood cells in Alsever's solution (MOUSE RED BLOOD CELLS, 88R-M001, Fitzgerald Industries International, North Acton, MA, USA). These erythrocytes were suspended in PBS (hematocrit of approximately $1 \%)$ and treated with ADNT $(0.1 \mathrm{mmol} / \mathrm{mL}, 30$ minutes before $)$ or phosphate buffered saline solution (PBS), in the presence of various BthTx-II concentrations $(5-80 \mu \mathrm{g} / \mathrm{mL})$ plus $1.5 \mathrm{mM}$ of lecithin, at $37^{\circ} \mathrm{C}$ for $60 \mathrm{~min}$ in a Dubnoff shaking apparatus. Aliquots were withdrawn after one hour, centrifuged and assayed for $\mathrm{Hb}$ in the supernatant. $\mathrm{Hb}$ concentration was determined spectrophotometrically at $540 \mathrm{~nm}$.

\subsubsection{Lipid peroxidation of membrane.}

Erythrocyte membrane lipid peroxidation were carried out with erythrocyte's suspension containing $0.5 \mathrm{mM}$ of Hemoglobin $(\mathrm{Hb})$ treated with ADNT $(0.1 \mathrm{mmol} / \mathrm{mL}, 30$ minutes before) or not, in the presence of various sPLA2 concentration $(5-80 \mu \mathrm{g} / \mathrm{mL})$ with $1.5 \mathrm{mM}$ of lecithin. After $60 \mathrm{~min}$ at $37^{\circ} \mathrm{C}, 0.5 \mathrm{ml}$ of $25 \%$ trichloroacetic acid (TCA) was added to $1 \mathrm{ml}$ of suspension and the mixture was centrifuged at $1100 \mathrm{~g}$ for $5 \mathrm{~min}$. To $1 \mathrm{ml}$ of the resulting supernatant, $1 \mathrm{ml}$ of $1 \%$ thiobarbituric acid (TBA) in $0.05 \mathrm{M} \mathrm{NaOH}$ was added, followed by boiling for $15 \mathrm{~min}$. The formation of TBA-reactive substances (TBARS) was used as a measure of lipid peroxidation. TBARS concentration was determined using an $\varepsilon$ of $156 \mathrm{mM}-1 \mathrm{~cm}^{-1}$ at $532 \mathrm{~nm}$.

\subsubsection{Hb oxidation}

For $\mathrm{Hb}$ oxidation, distinct concentrations of BthTx-II were added to $40 \mu \mathrm{M}$ of hemoglobin previously incubated with ADNT (in $0.1 \mathrm{M}$ phosphate buffer, $\mathrm{pH}$ 7.4). The extent of $\mathrm{Hb}$ oxidation was measured after 15 min incubation at 37 ॰C using a Hitachi U2000 spectrophotometer. Relative proportions of oxyHb, metahemoglobin (metHb) and hemichrome (Hemi) were calculated using following the equations.

Equation: $\quad[\mathrm{OxyHb}]=119 \mathrm{~A} 577-39 \mathrm{~A} 630-89 \mathrm{~A} 630$

$[\mathrm{MetHb}]=28 \mathrm{~A} 577+307 \mathrm{~A} 630-55 \mathrm{~A} 560$

$[$ Hemi $]=-133 \mathrm{~A} 577-114 \mathrm{~A} 630+233 \mathrm{~A} 560$

\subsubsection{Free Thiol measurement.}

Free thiol content was determined using MET 5053 (Total Thiol Assay Kit (Colorimetric), Cell Biolabs, Inc., San Diego, CA, USA), according to manufacturer's guidelines. Aliquots with $50 \mu \mathrm{L}$ of $40 \%$ suspension of red blood cell in PBS were used. The GSH concentration was expressed in nmols/mg protein.

\subsection{Statistical Analysis.}


Results are reported as the means \pm SD of replicated experiments. The significance of differences between means was assessed by an analysis of variance (ANOVA) followed by Dunnett's test when several experimental groups were compared to the control group. The confidence limit for significance was $5 \%$.

\section{Results}

\subsection{Edema and biochemical profile of BthTx-II without any treatment.}

Figure 01 shows the sPLA2-induced edema of Bothrops jararacussu sPLA2 (BthTx-II). The purified BhTx-II reveals a typical acute edema profile, which in 30 minutes exhibits a swelling value of $0.20 \pm 0.046\left(n=5\right.$ and $\left.{ }^{*} P \leq 0.05\right)$ followed by a maximum edema peak in 60 minutes with a swelling value of $0.24 \pm 0.033\left(n=5\right.$, and $\left.{ }^{*} P \leq 0.05\right)$. At 240 minutes, the edema value is close to the saline-induced edema, as shown in the graph of Figure 1A.

At 30 minutes after the BthTx-II injection, COX-2 levels were evaluated and results highlight an increased level of COX-2, which was $28.56 \pm 1.21 \mathrm{ng} / \mathrm{mL}\left(\mathrm{n}=5\right.$, and $\left.{ }^{*} \mathrm{P} \leq 0.05\right)$.In contrast, the COX-2 expression obtained at 60 minutes, which means at the edema peak, achieves values close to negative control, which was $9.65 \pm 2,07 \mathrm{ng} / \mathrm{mL}\left(\mathrm{n}=5\right.$, and ${ }^{*} \mathrm{P} \leq$ 0.05) (Figure 1B). Evaluation of PGE2 values were performed revealing a high level at 30 minutes, however this concentration decreases close to the negative control values until edema process ends (Figure 1C). These data suggest the Araquidonic acid (AA) mobilization and the consequent increase of COX-2 and PGE-2 expression occur before the edema peak induced by BthTx-II.

Malondialdehyde (MDA) was selected as a marker to evaluate lipid peroxidation and figure 1D shows the MDA concentration values at 30 minutes and 60 minutes were $27.3 \pm$ $1.43 \mathrm{nmols} / \mathrm{mg}\left(\mathrm{n}=5\right.$, and $\left.{ }^{*} \mathrm{P} \leq 0.05\right)$ and $18.8 \pm 1.83 \mathrm{nmols} / \mathrm{mg}\left(\mathrm{n}=5\right.$, and $\left.{ }^{*} \mathrm{P} \leq 0.05\right)$, respectively. Another parameter that was selected for this work was the proteins carbonylation increasing, which indicates the decrease in the antioxidant capacity. Figure 1E reveals a transient increase in the concentration of proteins carbonylation concentration, however, with values close to the observed in control.

The amount of free thiol was demonstrated in figure $1 \mathrm{~F}$, exhibiting the free thiols numbers for the treatment with saline, at 30, 60 and 240 minutes was approximately 27 mmols/ mg of protein throughout the observation period. In the case of BthTx-II treatment, a significant decay in the free thiols concentration is observed over the times of 30 and 60 minutes and the concentrations of thiol return close to the values observed in the control at 240 minutes. Thiol content quantity that exists in small molecules such as free cysteine, glutathione, cysteine residues, and peroxyredoxins are included in the family of heme-free thiol peroxidase as well as in glutathione peroxidases (GPx). Thus, monitoring free thiol amount present in a sample can indirectly measure the thiol-dependent antioxidant system recruitment.

\subsection{Edema and Myonecrosis profiles of BthTx-II under treatment with PRXs in-} hibitors.

Figure 2A displays the edema profile induced by sPLA2 BthTx-II, which was analyzed in time intervals from 0 to 480 minutes. In the period of 30 and 60 minutes (recent phase) we observed an increase in edema values, which reached the maximum value at 60 minutes. In addition, figure 2A shows that after 180 minutes a marked decrease in edema is observed in the late phase. 300 minutes after edema induction, the values decreased close to the negative control levels. 


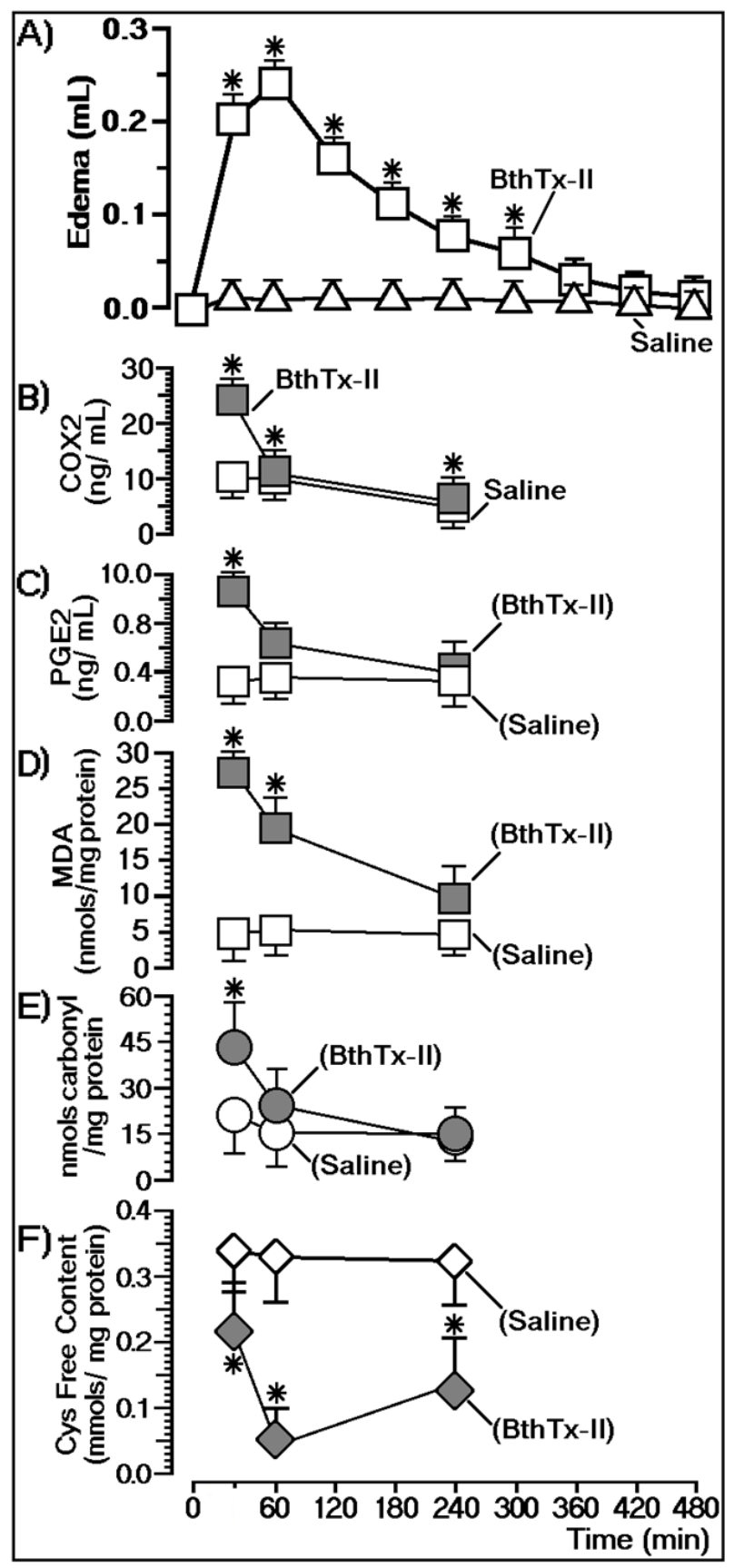

Figure 1. BthTx-II edema profile and the COX-2, PGE2, MDA, carbonylation and cys free content. Figure 1A shows the induced edema profile expressed as paw swelling volume $(\mathrm{mL})$ measured for 480 minutes. All observations were made using an $\mathrm{n}=5$ and the asterisk indicates ${ }^{*} \mathrm{P} \leq 0.05$ of five observations. Figure $1 \mathrm{~B}$ exhibits 5 panels $(1 \mathrm{~B}-1 \mathrm{~F})$, which were obtained from the animals' blood treated with BthTx-II in the same edema test conditions. and the blood collection at the intervals indicated from an $n=5$ animals for each collection time, adding a total of 15 animals. Samples were collected at 30, 60 and 240 minutes to evaluate at each point the COX2, PGE2, MDA, carbonylation of proteins and free thiols.

The edema profile induced by BthTx-II in animals treated with ADNT reveals a markedly increased edema in all the time intervals, mainly after 120 minutes. Bothrops jararacussu sPLA2 induced swelling with values of $0.26 \pm 0.021 \mathrm{~mL}(\mathrm{n}=5)$ and $0.28 \pm 0.067$ $\mathrm{mL}(\mathrm{n}=5)$ at 30 and 60 minutes respectively while the swelling values induced by BthTx- 
II for untreated animals showed swelling values of $0.20 \pm 0.046 \mathrm{~mL}\left(\mathrm{n}=5,{ }^{*} \mathrm{P} \leq 0.05\right)$ and the highest peak at 60 minutes of the edema experiment with a swelling value of $0.24 \pm$ $0.033 \mathrm{~mL}\left(\mathrm{n}=5,{ }^{*} \mathrm{P} \leq 0.05\right)$. According to figure $2 \mathrm{~A}$, the values of edema induced by BthTxII previously treated with ADNT do not show a swelling decreasing tendency even after 480 minutes and showed a value of $0.25 \pm 0.028 \mathrm{~mL} / \mathrm{mg}\left(\mathrm{n}=5,{ }^{*} \mathrm{P} \leq 0.05\right)$. On the other hand, the swelling induced by BthTx-II in the untreated group the edema value decreases until to the same value found in the control.

Furthermore, figure $2 \mathrm{~A}$ reveals that animals treated with the inhibitor ConA, which is a covalent inhibitor of peroxiredoxins II and I did not affect the edematogenic activity of sPLA2 BthTx-II and all swelling values were statistically equal edema values of untreated animals (Figure 1A). Besides, in addition, the edematogenic effect induced by BthTx-II in a group of animals previously treated with MJ 33 inhibitor, which is a potent inhibitor of Prdx6 PLA2 activity, significantly decreased the edema values induced by untreatedgroup. MJ 33 administration was able to reduce edema values at 30,60 and 120 minutes besides to reduce edema peak of BthTx-II. Figure 2B exhibits BthTx-II myotoxic activity profile of untreated and treated groups with ADNT, ConA and MJ33.CK values in untreated animals were $315 \pm 18.34 \mathrm{U} / \mathrm{L}\left(\mathrm{n}=5\right.$, and $\left.{ }^{*} \mathrm{P} \leq 0.05\right)$ while animals treated with ConA did not affect BthTx-II myotoxic effect. On the other hand, the treatment with ADNT, reveals that BthTx-II features a myotoxic activity two times higher than sPLA2 in untreated animals. These results suggest that the antioxidant thiol-dependent system also plays a crucial role controlling the cellular oxidative stress levels. Moreover, conA treatment had no significant effect on BthTx-II myotoxic activity. Nevertheless, results from animals treated with MJ33 reduced the myotoxic capacity of BthTx-II revealing a CK value of $235 \pm 11.24 \mathrm{U} / \mathrm{L}\left(\mathrm{n}=5\right.$, and $\left.{ }^{*} \mathrm{P} \leq 0.05\right)$ highlighting a decrease of approximately $25 \%$ of the myotoxic activity.

\subsection{Hemolysis Mouse Red Blood Cells and ADNT.}

BthTx-II hemolytic activity exhibits a dependent dose profile with doses of 5, 10, 20, 40 and 80 micrograms/mL. According to figure 3A, at $80 \mu \mathrm{g} / \mathrm{mL}$ was observed the highest percentage values of hemolysis $\left(93.6 \% \pm 3, \mathrm{n}=12\right.$, and $\left.{ }^{*} \mathrm{P} \leq 0.05\right)$. This value was obtained with 80 micrograms/ mL for red blood cells treated with PBS. However, animals treated with ADNT revealed the highest percentage of hemolysis at 40 micrograms/ mL. Figure 3B displays the levels of TBARS, which increased in a dose-dependent manner, reaching a maximum of $23.6 \pm 2.7$ nmols/ $\mathrm{mg}$ of protein $\left(\mathrm{n}=12\right.$, and $\left.{ }^{*} \mathrm{P} \leq 0,05\right)$ in the control group (PBS).). Moreover, in the ADNT treatment, TBARS concentration reached maximum values with a BthTx-II concentration of 80 micrograms/ mL. Figure 3C displays the methemoglobin concentration which also followed the same dose-dependent trend, reaching the maximum peak at $80 \mathrm{micrograms} / \mathrm{mL}$ of BthTx-II with a value of $19.2 \pm 0.6 \mathrm{nmol} / \mathrm{pro}-$ tein $(\mathrm{mg})\left(\mathrm{n}=12\right.$, and $\left.{ }^{*} \mathrm{P} \leq 0.05\right)$ in the PBS treatment. Higher values were observed in the treatment with ADNT, which achieved approximately $35 \mathrm{nmol} /$ protein $(\mathrm{mg})$. The free thiol levels were also evaluated, revealing a dose-dependent decay of these molecules (figure 3D). These assays highlight most samples incubated with ADNT in comparison to PBS showed results considerably higher with the incubation of PRXs inhibitor. 


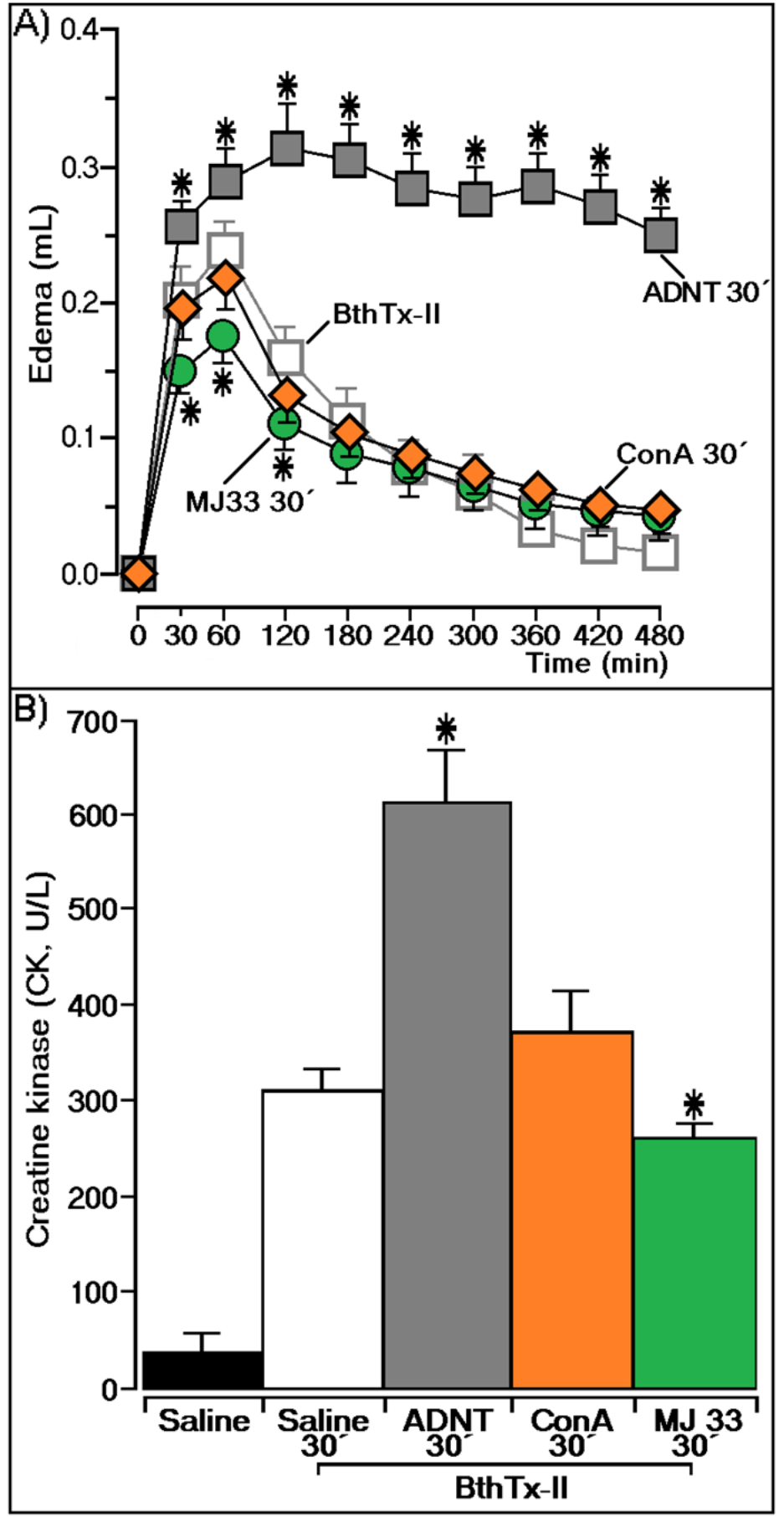

Figure 2. Paw edema assay with animals treated with peroxiredoxins inhibitors and creatine kinase values of each treatment. Figure $1 \mathrm{~A}$ shows the paw edema evaluation treated with ADNT, ConA and MJ33, which are PRXs inhibitors. These compounds were injected 30 minutes before sPLA2 application. The results are expressed as means \pm standard deviation $(n=5)$. Significant differences are marked with asterisk indicates ${ }^{*} P \leq 0.05$ of five observations. Figure $1 \mathrm{~B}$ exhibits the myotoxicity of the groups treated with these inhibitors, which were applied 30 minutes before injection of sPLA2 in the gastrocnemius muscle. The results are expressed as means \pm standard deviation $(n=5)$. Significant differences are marked with $\left({ }^{*}\right)$ asterisk indicates ${ }^{*} \mathrm{P} \leq 0.05$ of five observations. 


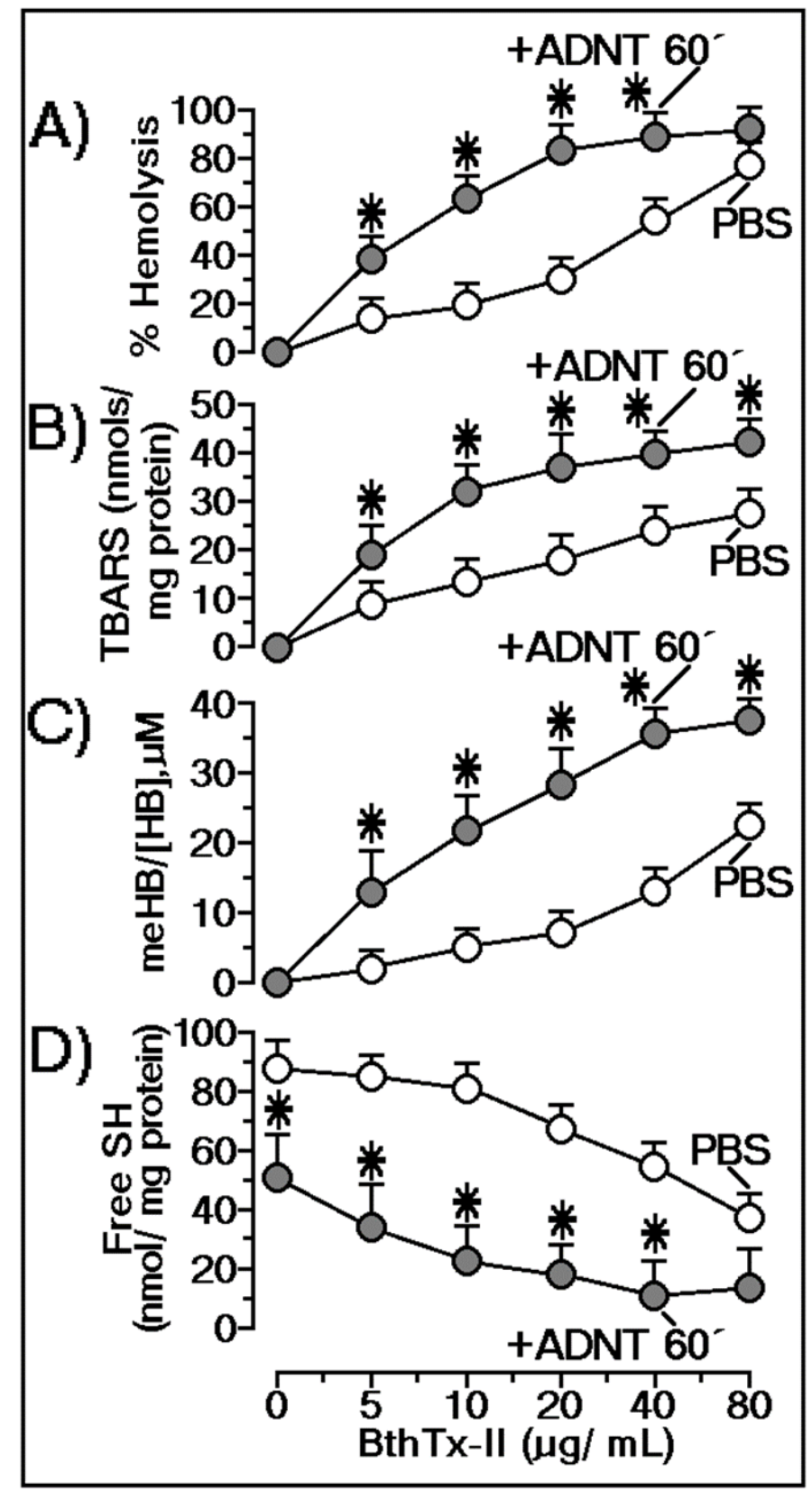

Figure 3. Dose response effect profile of different BthTx-II concentration on the mouse indirect hemolysis in red blood cells treated with Adenanthin (Adn) or not. Figure 7A and 7B exhibit an indirect hemolysis, which follows a dose response curve proportional to BthTx-II concentration. Both figures reveal this indirect homolysis occurs through the TBA increasing, which is an indirect form of lipid peroxidation. TBA reactive substances (TBARS) concentration was measured at $532 \mathrm{~nm}$. Figure 3C shows that methemoglobin $(\mathrm{MetHb})$ is formed by the reversible oxidation of heme iron to the ferric state $(\mathrm{Fe} 3+)$. Normally, a small amount of methemoglobin is continuously formed by oxidation of iron during normal oxygenation and deoxygenation of hemoglobin. However, in the presence of increasing doses of BthTx-II occurs an MetHb rise, which is observed due to the increased TBA and indirect hemolysis. Aliquots of ADNT incubated with mouse erythrocytes were more susceptible to indirect hemolysis. The results are expressed as means \pm standard deviation $(n=5)$. In panel $3 \mathrm{D}$ reveal the possible participation of the dependent thiols enzymes in the protection of the oxidative stress, a process significantly increased due to the Bothrops jaracussu sPLA2 activity 


\section{Discussion.}

The role of oxidative stress on the course and complications of pathological events arising from ophidic accidents has already evidenced, however poorly understood. Biomedical, clinical and laboratory studies indicate the envenomation leads to a systemic and pronounced oxidative stress measured on patients blood [28-30]. Besides that, some investigations indicate the toxicity induced by snake venom increases the reactive oxygen species and hidrogen peroxide, which is an essential element on the pharmacological process induced by snake venom and its toxins. Hence, the production of these molecules can possibly be related to the increasing cytotoxic and necrotizing snake venom potential [3134, 8, 3].

The findings presented in this work confirm several studies that report the essential role of oxidative stress during the pathophysiological action of snake venom. Furthermore, our investigation focused on mainly on paw edema, a crucial model for the acute inflammation study, which is a common condition after the bite of poisonous snakes. Data presented in figure 1 suggest essential aspects in the evolution of the course and edema induced by BthTx-II, which seems to be the relationship between AA production and the increase in cellular oxidative stress evidenced by the high concentrations of MDA and protein carbonylation. In addition, the biochemical results indicate that the AA pathway metabolites and markers related to oxidative stress appear before the edema peaks (Figure $1, a, b$ and $c)$. Besides that, the lasting phase of edema resolution involves a significant free thiols mobilization during the first 120 minutes, exhibiting the greatest peak at 60 minutes (figure $1 \mathrm{~F}$ ) and at 240 minutes the thiols levels were significantly lower than at 30 minutes.

Research reveals during the inflammatory process it is generated a significant increase in cellular oxidative stress, besides and that the thiols concentration in cells plays a fundamental role on the thiol-dependent antioxidant enzymes maintenance to control cellular oxidative stress [35] and mainly enzymes such as GHS [36]. Our results also show the thiols mobilization, which displayed an important role to resolute the acute inflammation induced by BthTx-II.

Data presented in figure 2 suggests that the thiol-dependent antioxidant system would also play a significant role controlling the oxidative stress and decreasing it, helping to control and decrease paw edema. In this process peroxiredoxins could play a crucial role. in the oxidative stress control related to AA and its metabolites. Their activity can also support cells to regulate the AA and its derivatives oxidation and when this system fails, markers related to lipid liporoxidation may appear in the circulation [37-40]. In contrast to catalases and superoxide dismutases, peroxidases use a thiol-based reaction mechanism to detoxify hydroperoxides and their reduced state needs to be restored through the GSH / GR or Trx / TrxR system under NADPH consumption (or in a special case, $\mathrm{NADH})[41,22]$.

Our research indicates that animals treated with ADNT, ConA and MJ33 inhibitors show promising results. ADNT is reported in the literature as a non-specific inhibitor for peroxiredoxin I / II, which can also inhibit several free thiols including thioredoxin and thioredoxin reductase besides to reduce the glutathione levels in erythrocytes through glutathione inhibition $[42,43,44]$. On the other hand, ConA is being sold as a covalent inhibitor of peroxiredoxin II, however, other reports show that this compound can also inhibit peroxiredoxin I. The results in Figure 2A reveal that animals pretreated with Con A did not alter the edema profile induced by BthTx -II. Contrasting, animals pretreated with ADNT developed a persistent edema over 360 minutes, which only started to show a slight decrease in swelling after 420 minutes. Moreover, these results suggest that peroxiredoxin I or II alone would not play a crucial role controlling oxidative stress induced by sPLA2 BthTx-II. ConA results support this idea once although itwould be a specific inhibitor for peroxiredoxin II and I, it could be part of the thiol-dependent enzymes chain that are crucial to control the edema process.

Several studies also state that PRXs present a dual role which would be the antioxidant role besides to act as a chaperone in its dimeric form. As a chaperone, PRXs are 
crucial to assist denatured proteins $[45,46]$ and Trxs display an essential role in the transition from chaperone function to peroxidase [46]. Studies describe the Trxs system, as a system that is composed by NADPH, thioredoxin reductase (TrxR), and thioredoxin and it is a key-antioxidant-system crucial to defend cells against oxidative stress. This activity occurs through its disulfide reductase activity regulating protein dithiol/disulfide balance. Additionally, this system together with the glutathione-glutaredoxin (Grx) system, (NADPH, glutathione reductase, GSH and Grx) control the cellular redox environment. Other investigations also show that ADNT acts as a generic inhibitor of several thiol-dependent enzymes including Trxs, TrxR and GSH $[43,44]$. Thus, these results with ADNT and Con A suggest that the dependent thiol enzymes could assume a yet undescribed and unknown role of an important source of antioxidant agents controlling the acute inflammation and myotoxicity induced by BthTx-II.

PRDX6 belongs to PRDXs and is the only bifunctional enzyme that acts as glutathione peroxidase and phospholipase A2 (PLA2). As a PLA2, this enzyme can hydrolyze phospholipids through $\mathrm{H} 2 \mathrm{O} 2$, short chain hydroperoxides and phospholipids reduction [47]. Furthermore, PRDX6 also regulates several enzymes from NADPH oxidases (NOXs) family that play a role regulating the activation of the cell oxidative stress, proinflammatory pathways and routes related. Studies with MJ33 inhibitor, highlight PRDX6 inhibition leads to a remarkable decrease in inflammatory activity [48, 49], however, it had never been observed in the acute edema induced by BthTx-II. In this study figure 2A clearly reveals animals previously treated with MJ33 significantly decreased BthTx-II-induced edema. Besides that, our results suggest that AA generation is intricately linked to increased oxidative stress, since it is a strong regulator of NOXs activity [8,50]. Furthermore, PRDX6 plays an important role mediating edema early stages through another route that would reinforce the oxidative stress during edema.

Further investigations reveal that extracts from plants and their antioxidant components can neutralize the myotoxic effects induced by certain toxins such as BthTx-II [51, 52]. This enzyme is known to causes evident muscle tissue damage characterized by distinct ultra-structural modifications, which can be measured through the CK levels. Nevertheless, many studies have shown that natural compounds are able to induce a strong decay of myotoxic activity and the reason for this would be the interaction of compounds with myotoxic sPLA2 [53].

All these studies do not show another possible action of the compounds which is to strengthen antioxidant defenses and their importance on myotoxicity induced by sPLA2 such as BthTx-II. Our results display that myotoxicity induced by BthTx-II in groups of animals treated with ADNT shows that the average $\mathrm{CK}$ values were two times higher than the group that received saline (Figure 2-b). These results reinforce the role of thiol-dependent enzymes as an essential agent in the myotoxicity control once their neutralization resulted in a significant increase in $\mathrm{CK}$ values. Moreover, treatment with Con A inhibitor shows that PRXs have not changed the myotoxic activity of BthTx-II. Despite the role of reactive oxygen species (ROS) in myotoxicity, this is not considered an important aggravating factor in this case. Studies highlight the increased oxidative stress is related to rise the myotoxicity and induces increased SOD, CAT and GPX [54, 55]. Even though MJ33 showed a statistically significant decrease among the inhibitors tested, in practice, its values were remarkably close to the group of animals that received only saline.

Figure 3 displays that erythrocytes treated with ADNT are highly susceptible to the hemolytic action of BthTx-II and this is since snake venoms and their fractions such as PLA2 can cause hemolysis by increasing cellular oxidative stress [56, 57]. In addition, ADNT activity on these already well-known antioxidants thiol-dependent system and their inhibition effects reveal their crucial participation in the erythrocytes protection against the effects of oxidative stress by these proteins [58, 43, 59, 14]. It is well known that snake venoms and their fractions such as PLA2 can lead to hemolysis due to the increase of cellular oxidative stress $[56,57]$ and PRXs have an essential role in the erythrocytes protection against the oxidative stress effects induced by these proteins $[58,43,59,14]$. 
Our results exhibited in the figure 2 that thiol-dependent enzymes are involved in the cell protection against the edema and myotoxicity induced by BthTx-II, besides, PRXs I and II are not the unique important elements. Moreover, our hemolytic results emphasize that erythrocytes treated with ADNT are highly susceptible to the BthTx-II hemolytic effect, highlighting the participation of PRXs I and II in this process.

\section{Conclusions}

BthTx-II is a myotoxic and edematogenic phospholipase A2 (PLA2) D49, which induces an increase in peak expression of cyclooxygenase -2 (COX-2), prostaglandin E2 (PGE2), a peak concentration of malondialdehyde (MDA) and protein carbonylation in the early phase of BthTx-II-induced edema. The late phase and the decrease in edema induced by this protein could involve the thiol-dependent antioxidants mobilization. Thus, edema and other activities such as myotoxicity and erythrocyte hemolysis, strongly include an increase in cellular oxidative stress in the early phase. In this way, this study aimed to evaluate the peroxiredoxins enzymatic system, which is one of the most essential defenses to neutralize lipid peroxidation. Lipid peroxidation is one of the main products found in patients bitten by Bothrops sp and Crotalus durissus sp snakes as indicated in the little available biomedical and clinical literature. In this work we investigated the action of commercial inhibitors such as Adenantine (ADNT) which is an inhibitor of peroxyredoxin 1 (PRDX1) and peroxyeredoxin 2 (PRDX2), Conoidin A (Con A) which is a covalent inhibitor of peroxyeredoxin II and MJ33 which is an inhibitor of peroxyeredoxin 6 . All these inhibitors were used at the same concentration and injected 30 minutes before sPLA2 application $(0.5 \mathrm{mg} / \mathrm{mL})$. ADNT boosted the edematogenic and myotoxic effects of BthTx-II compared to BthTx-II without any prior treatment. In addition, ADNT also induced a significant increase in BthTx-II-induced hemolysis and involved an increase in cellular oxidative stress, as in edema and myotoxicity. The results with the peroxiredoxin 6 inhibitor suggest that calcium-independent cytosolic PLA2 plays a relevant role in initiating the inflammatory and myotoxic activities of BhTx-II. Furthermore, the Con A treatment results suggest that PRDXs would not be the only proteins involved in protecting against BthTx-II-induced oxidative stress. However other enzymes such as thioredoxin $(\mathrm{TXN})$, thioredoxin reductase (TXNRD) and other glutathione (GSH)-related antioxidant defenses could play an essential role controlling and defining the end of edema on the late phase of PLA2 BthTx-II-induced process.

6. Patents: No patents, nothing to declare for this section.

Supplementary Materials: No supplemental materials have been posted in this article.

\section{Author Contributions:}

Prof. Toyama: Conceptualization; formal analysis; visualization; project administration; funding acquisition, writing-review and editing and supervision.

Caroline R. C. Costa (Doctoral student), Mariana Novo Belchor (Doctoral student ), Adeilso Bispo dos Santos Junior (Master student ), Laila Lucyane Ferreira de Moraes (Master student), Airam Roggero dos Santos Silva (Master student): methodology, software analysis, validation, formal analyss, experiment execution e reports of experiments, original draft preparation.

Caroline R. C. Costa (Doctoral student), Mariana Novo Belchor (Doctoral student ): review and editing and grammar correction.

Marcos A. Oliveira: original draft preparation, supervision, Conceptualization and investigation. 
All authors have read and agreed to the published version of the manuscript." Please refer to the CRediT taxonomy for the explanation of the term. Authorship should be limited to authors who have substantially contributed to the reported work.

All authors have read the paper and approved its submission and they will be receiving it as final copies.

Funding: Please add: Este trabalho teve suporte financeiro da FAPESP PROC. No. 2017/20291-0/ AUXILIO PESQ. e da FAPESP PROC. No. 2017/19942-7/ AUXILIO PESQ. e o autor é pesquisador PQ2 CNPq (CNPQ 2019 304153/2019-2).

Institutional Review Board Statement: The study was conducted according to the guidelines and that validated the studies In vivo experiments were performed according to institutional rules and were approved by the ethics committee of UNESP, number 008-CEUA"

Informed Consent Statement: “Not applicable” for studies not involving humans.

Data Availability Statement: All data in the paper followed all current analyses and are original.

Acknowledgments: Agradecimentos a todos os colaboradores que se mantiveram firmes e resolutos, memo com a pandemia do COVID-19. We are very thankful to the Fundação de Amparo à Pesquisa do Estado de São Paulo, which is one of the main funding agencies for scientific and technological research in the country and which supports several research initiatives in the state of São Paulo. We thank Conselho Nacional de Desenvolvimento Científico e Tecnológico of the government of the Federative Republic of Brazil for its support of the productivity fellows. To our university (Unesp - São Paulo State University) for the institutional support to our research efforts.

Conflicts of Interest: The authors declare no conflict of interest

\section{Appendix A}

Not applicable

\section{Appendix B}

Not applicable.

\section{References}

1. Santhosh, M.S.; Sundaram, M.S.; Sunitha, K.; Kemparaju, K.; Girish, K.S. Viper venom-induced oxidative stress and activation of inflammatory cytokines: a therapeutic approach for overlooked issues of snakebite management. Inflamm Res. 2013, 62(7), 721-731.

2. Sharma, R.D.; Katkar, G.D.; Sundaram, M.S.; Swethakumar, B.; Girish, K.S.; Kemparaju, K. 2017. Melatonin inhibits snake venom and antivenom induced oxidative stress and augments treatment efficacy. Acta Tropica, 2017, 169, 14-25.

3. Dong, D.; Deng, Z.; Yan, Z.; Mao, W.; Yi, J.; Song, M.; Li, Q.; Chen, J.; Chen, Q.; Liu, L.; Wang, X.; Huang, X.; Wang, W. Oxidative stress and antioxidant defense in detoxification systems of snake venom-induced toxicity. J. Venom. Anim. Toxins incl. Trop. Dis. 2020, 26, e20200053.4.

4. Morais, V.M.; Massaldi, H. Snake antivenoms: adverse reactions and production technology. J. Venom. Anim. Toxins incl. Trop. Dis., 2009, 15(1), 2-18.

5. 5. de Silva, H.A.; Ryan, N.M.; de Silva, H.J. Adverse reactions to snake antivenom, and their prevention and treatment. Br. J. Clin. Pharmacol., 2016, 81(3), 446-452.

6. Pereañez, J.A.; Lobo-Echeverri, T.; Rojano, B.; Vargas, L.; Fernandez, M.; Gaviria, C.A.; Vitelbina, N. Correlation of the inhibitory activity of phospholipase A2 snake venom and the antioxidant activity of Colombian plant extracts. Rev. bras. Farmacognosia, 2010, 20( 6 ), 910-916.

7. Mukhopadhyay, P.; Mishra, R.; Mukherjee, D.; Mishra, R.; Kar, M. Snakebite mediated acute kidney injury, prognostic predictors, oxidative and carbonyl stress: A prospective study. Indian J Nephrol. 2016, 26(6), 427433. 
8. Toyama, M.H.; Costa, C.R.C.; Belchor, M.N.; Novaes, D.P.; de Oliveira, M.A.; Ie, R.; Gaeta, H.H.; Toyama, D.O. Edema Induced by sPLA2 from Crotalus durissus terrificus Involves PLC and PKC Signaling, Activation of cPLA2, and Oxidative Stress, IntechOpen, 2019. DOI: 10.5772/intechopen.80848.

9. Valko, M.; Leibfritz, D.; Moncol, J.; Cronin, M.T.; Mazur, M.; Telser, J. Free radicals and antioxidants in normal physiological functions and human disease. Int J Biochem Cell Biol., 2006, 39(1), 44-84.

10. Bedard, K.,Krause, K-H. The NOX Family of ROS-Generating NADPH Oxidases: Physiology and Pathophysiology. Physiological Reviews 2007, 87(1), 245-313.

11. Repetto, M.G.; Ferrarotti, N.F.; Boveris, A. The involvement of transition metal ions on iron-dependent lipid peroxidation. Arch Toxicol. 2010, 84(4), 255-262.

12. Maraldi, T. "Natural Compounds as Modulators of NADPH Oxidases", Oxidative Medicine and Cellular Longevity, vol. 2013, Article ID 271602, 10,

13. Sayre, L.M.; Lin, D.; Yuan, Q.; Zhu, X.; Tang, X. 2006. Protein adducts generated from products of lipid oxidation: focus on HNE and one. Drug Metab Rev. 2006, 38(4), 651-675.

14. Lang, F.; Abed, M.; Lang, E.; Föller, M. Oxidative stress and suicidal erythrocyte death. Antioxid Redox Signal. 2014, 21(1), 138-153.

15. Schaur, R.J.; Siems, W.; Bresgen, N.; Eckl, P.M. 4-Hydroxy-nonenal-A Bioactive Lipid Peroxidation Product. Biomolecules, 2015, 5(4), 2247-2337.

16. Petersen, R.C. Free-radicals and advanced chemistries involved in cell membrane organization influence oxygen diffusion and pathology treatment. AIMS Biophys., 2017, 4(2),240-283.

17. Ayala, A.; Muñoz, M.F.; Argüelles, S. "Lipid Peroxidation: Production, Metabolism, and Signaling Mechanisms of Malondialdehyde and 4-Hydroxy-2-Nonenal", Oxidative Medicine and Cellular Longevity, 2014, 31.

18. Lu, J.; Holmgren, A. The thioredoxin antioxidant system. Free Radic Biol Med., 2014, 66,75-87.

19. Aoyama, K.; Nakaki, T. Glutathione in Cellular Redox Homeostasis: Association with the Excitatory Amino Acid Carrier 1 (EAAC1). Molecules (Basel, Switzerland), 2015, 20(5), 8742-8758.

20. Knoops, B.; Argyropoulou, V.; Becker, S.; Ferté, L.; Kuznetsov, O. Multiple Roles of Peroxiredoxins in Inflammation. Mol Cells. 2016, 39(1), 60-4.21

21. Rhee, S.G.; Woo, H.A.; Kang, D. The Role of Peroxiredoxins in the Transduction of $\mathrm{H}(2) \mathrm{O}(2)$ Signals. Antioxid Redox Signal., 2018, 28(7), 537-557.

22. Ulrich, K.; Jakob, U. The role of thiols in antioxidant systems. Free Radic Biol Med., 2019, 140,14-27.

23. Arevalo, J. A.; Vázquez-Medina, J. P. The Role of Peroxiredoxin 6 in Cell Signaling. Antioxidants (Basel, Switzerland), 2018, 7(12), 172.

24. Fisher, A.B. Antioxidants Special Issue: Peroxiredoxin 6 as a Unique Member of the Peroxiredoxin Family. Antioxidants (Basel), 2019, 8(4),107.

25. Hou, J.K.; Huang, Y.; He, W.; Yan, Z.W.; Fan, L.; Liu, M.H.; Xiao, W.L.; Sun, H.D.; Chen, G.Q. Adenanthin targets peroxiredoxin I/II to kill hepatocellular carcinoma cells. Cell Death Dis., 2014, 5(9),e1400.

26. Hu, J.; Li, X.; Tian, W.; Lu, Y.; Xu, Y.; Wang, F.; Qin, W.; Ma, X.; Puno, P.T.; Xiong, W. Adenanthin, a Natural ent-Kaurane Diterpenoid Isolated from the Herb Isodon adenantha Inhibits Adipogenesis and the Development of Obesity by Regulation of ROS. Molecules, 2019, 24(1),158.

27. Nguyen, J.B.; Pool, C.D.; Wong, C.Y.; Treger, R.S.; Williams, D.L.; Cappello, M.; Lea, W.A.; Simeonov, A.; Vermeire, J.J.; Modis, Y. Peroxiredoxin-1 from the human hookworm Ancylostoma ceylanicum forms a stable oxidized decamer and is covalently inhibited by conoidin A. Chem Biol., 2013, 20(8), 991-1001.

28. Katkar, G.D.; Sundaram, M.S.; Hemshekhar, M.; Sharma, D.R.; Santhosh, M.S.; Sunitha, K.; Rangappa, K.S.; Girish, K.S.; Kemparaju, K. Melatonin alleviates Echis carinatus venom-induced toxicities by modulating inflammatory mediators and oxidative stress. J Pineal Res., 2014, 56(3), 295-312.

29. Sunitha, K.; Hemshekhar, M.; Thushara, R.M.; Santhosh, M.S.; Sundaram, M.S.; Kemparaju, K.; Girish, K.S. Inflammation and oxidative stress in viper bite: an insight within and beyond. Toxicon, 2015, 98, 89-97.

30. Strapazzon, J.O.; Parisotto, E.B.; Moratelli, A.M.; Garlet, T.R.; Bastos, J.; Zimermann, I.R.; Zanin, M.; Fagundez, R.; Lino, M.R.O.; Frode, T.S.; Wilhelm Filho, D. Systemic oxidative stress in victims of Bothrops snakebites. J. Appl. Biomed, 2015, 13, 161-167. 
31. Sebastin Santhosh, M.; Hemshekhar, M.; Thushara, R.M.; Devaraja, S.; Kemparaju, K.; Girish, K.S. Vipera russelli venom-induced oxidative stress and hematological alterations: amelioration by crocin a dietary colorant. Cell Biochem Funct., 2013, 31(1), 41-50.

32. Al-Asmari, A.K.; Riyasdeen, A.; Al-Shahrani, M.H.; Islam, M. Snake venom causes apoptosis by increasing the reactive oxygen species in colorectal and breast cancer cell lines. Onco Targets Ther., 2006, ,6485-6498.

33. Meléndez-Martínez, D.; Muñoz, J.M.; Barraza-Garza, G.; Cruz-Peréz, M.S.; Gatica-Colima, A.; AlvarezParrilla, E.; Plenge-Tellechea, L.F. 2017. Rattlesnake Crotalus molossus nigrescens venom induces oxidative stress on human erythrocytes. J. Venom. Anim. Toxins incl. Trop. Dis., 2017, $23,24$.

34. Al-Sheikh, Y.A.; Ghneim, H.K.; Aljaser, F.S.; Aboul-Soud, M.A. Ascorbate ameliorates Echis coloratus venom-induced oxidative stress in human fibroblasts. EXP THER MED, 2017, 14, 703-713.

35. Santangelo F. Intracellular thiol concentration modulating inflammatory response: influence on the regulation of cell functions through cysteine prodrug approach. Curr Med Chem., 2003, 10(23),2599-2610.

36. Ghezzi P. Role of glutathione in immunity and inflammation in the lung. Int J Gen Med., 2011, 4,105-13.

37. Cordray, P.; Doyle, K.; Edes, K.; Moos, P.J.; Fitzpatrick, F.A. Oxidation of 2-Cys-peroxiredoxins by arachidonic acid peroxide metabolites of lipoxygenases and cyclooxygenase-2. J Biol Chem., 2007, 282(45), 32623-9.

38. Yoshida, Y.; Umeno, A.; Shichiri, M. Lipid peroxidation biomarkers for evaluating oxidative stress and assessing antioxidant capacity in vivo. J Clin Biochem Nutr., 2013, 52(1), 9-16.

39. Ademowo, O.S.; Dias, H.K.I.; Burton, D.G.A.; Griffiths, H.R. Lipid (per) oxidation in mitochondria: an emerging target in the ageing process? Biogerontology. 2017, 18(6), 859-879.

40. Dias, I.H.K.; Milic, I.; Heiss, C.; Ademowo, O.S.; Polidori, M.C.; Devitt, A.; Griffiths, H.R. Inflammation, Lipid (Per)oxidation, and Redox Regulation. Antioxid Redox Signal., 2020, 33(3), 166-190.

41. Poole, L.B. The basics of thiols and cysteines in redox biology and chemistry. Free Radic Biol Med., 2015, 80, 148-157.

42. Liu, C.X.; Yin, Q.Q.; Zhou, H.C.; Wu, Y.L.; Pu, J.X.; Xia, L.; Liu, W.; Huang, X.; Jiang, T.; Wu, M.X.; He, L.C.; Zhao, Y.X.; Wang, X.L.; Xiao, W.L.; Chen, H.Z.; Zhao, Q.; Zhou, A.W.; Wang, L.S.; Sun, H.D.; Chen, G.Q. Adenanthin targets peroxiredoxin I and II to induce differentiation of leukemic cells. Nat Chem Biol., 2012 8(5),486493.

43. Soethoudt, M.; Peskin, A.V.; Dickerhof, N.; Paton, L.N.; Pace, P.E.; Winterbourn, C.C. Interaction of adenanthin with glutathione and thiol enzymes: Selectivity for thioredoxin reductase and inhibition of peroxiredoxin recycling. Free Radic. Biol. Med., 2014, 77, 331-339.

44. Siernicka, M.; Winiarska, M.; Bajor, M.; Firczuk, M.; Muchowicz, A.; Bobrowicz, M.; Fauriat, C.; Golab, J.; Olive, D.; Zagozdzon R. Adenanthin, a new inhibitor of thiol-dependent antioxidant enzymes, impairs the effector functions of human natural killer cells. Immunology., 2015, 146(1),173-83.

45. Jang, H.H.; Lee, K.O.; Chi, Y.H.; Jung, B.G.; Park, S.K.; Park, J.H.; Lee, J.R.; Lee, S.S.; Moon, J.C.; Yun, J.W.; Choi, Y.O.; Kim, W.Y.; Kang, J.S.; Cheong, G.W.; Yun, D.J.; Rhee, S.G.; Cho, M.J.; Lee, S.Y. Two enzymes in one; two yeast peroxiredoxins display oxidative stress-dependent switching from a peroxidase to a molecular chaperone function. Cell, 2014, 117(5), 625-635.

46. An, B. C.; Lee, S. S.; Lee, E. M.; Lee, J. T.; Wi, S. G.; Jung, H. S.; Park, W.; Chung, B. Y. A new antioxidant with dual functions as a peroxidase and chaperone in Pseudomonas aeruginosa. Molecules and cells, 2010, 29(2), 145151.

47. Arriga, R.; Pacifici, F.; Capuani, B.; Coppola, A.; Orlandi, A.; Scioli, M.G.; Pastore, D.; Andreadi, A.; Sbraccia, P.; Tesauro, M.; Daniele, N.D.; Sconocchia, G.; Donadel, G.; Bellia, A.; Della-Morte, D.; Lauro, D. Peroxiredoxin 6 Is a Key Antioxidant Enzyme in Modulating the Link between Glycemic and Lipogenic Metabolism. Oxid Med Cell Longev. 2019.

48. Arevalo, J.A.; Vázquez-Medina, J.P. The Role of Peroxiredoxin 6 in Cell Signaling. Antioxidants (Basel). 2018, 7(12), 172.

49. Aron, B.; Fisher, A.B. Peroxiredoxin 6: A Bifunctional Enzyme with Glutathione Peroxidase and Phospholipase A2 Activities. Antioxid Redox Signal. 2011, 15(3), 831-844.

49. Fisher, A.B. The phospholipase A2 activity of peroxiredoxin 6. J Lipid Res., 2018. 59(7), 1132-1147.

50. Gonzalez-Perilli, L.; Prolo, C.; Álvarez, M.N. Arachidonic Acid and Nitroarachidonic: Effects on NADPH Oxidase Activity. Adv Exp Med Biol. , 2019, 1127, 85-95. 
51. Fernandes, R.S.; Costa, T.R.; Marcussi, S.; Bernardes, C.P.; Menaldo, D.L.; Rodriguéz Gonzaléz, I.I.; Pereira, P.S.; Soares, A.M. 2011. Neutralization of pharmacological and toxic activities of Bothrops jararacussu snake venom and isolated myotoxins by Serjania erecta methanolic extract and its fractions. J. Venom. Anim. Toxins incl. Trop. Dis, 2011, 2011, 17(1), 85-93.

52. Cardoso, F.F.; Soares, A.S.; Dreyer, T.R.; Cavalcante, W.L.G.; Pai, M.D.; Gallacci, M.; Fontes, M.R.M. Neutralization of a bothropic PLA2-like protein by caftaric acid, a novel potent inhibitor of ophidian myotoxicity. Biochimie 2020, 170, 163-171.

53. Arias, S.P.; de Jesús Rodríguez, B.; Lobo-Echeverri, T.; Ramos, R.S.; Hyslop, S.; Rangel, V. Effects of Two Fractions of Swietenia macrophylla and Catechin on Muscle Damage Induced by BothropsVenom and PLA 2 . Toxins (Basel). 2019, 11(1):40.

54. Uchiyama, S.; Tsukamoto, H.; Yoshimura, S.; Tamaki, T. Relationship between oxidative stress in muscle tissue and weight-lifting-induced muscle damage. Pflugers Arch., 2006, 452(1), 109-116.

55. Ábrigo, J.; Elorza, A.A.; Riedel, C.A.; Vilos, C.; Simon, F.; Cabrera, D.; Estrada, L.; Cabello-Verrugio, C. Role of Oxidative Stress as Key Regulator of Muscle Wasting during Cachexia. Oxid Med Cell Longev. 2018, 2063179.

56. Meléndez-Martínez, D.; Muñoz, J.M.; Barraza-Garza, G.; Cruz-Peréz, M.S.; Gatica-Colima, A.; AlvarezParrilla, E. Rattlesnake Crotalus molossus nigrescens venom induces oxidative stress on human erythrocytes. J. Venom. Anim. Toxins incl. Trop. Dis., 2017, 23, 24.

57. Tonello, F.; Rigoni, M. 2017. Cellular Mechanisms of Action of Snake Phospholipase A2 Toxins. In: Gopalakrishnakone P., Inagaki H., Vogel CW., Mukherjee A., Rahmy T. (eds) Snake Venoms. Toxinology 2017. pringer, Dordrecht.

58. Rocha, S.; Costa, E.; Coimbra, S.; Nascimento, H.; Catarino, C.; Rocha-Pereira, P.; Quintanilha, A.; Belo, L.; Santos-Silva, A. Linkage of cytosolic peroxiredoxin 2 to erythrocyte membrane imposed by hydrogen peroxide-induced oxidative stress. Blood Cells Mol Dis., 2009, 43(1), 68-73.

59. Mohanty, J.G.; Nagababu, E.; Rifkind, J.M. Red blood cell oxidative stress impairs oxygen delivery and induces red blood cell aging. Front Physiol 2014, 5, 84. 